EPJ Web of Conferences 59, 09001 (2013)

DOI: $10.1051 /$ epjconf/20135909001

(C) Owned by the authors, published by EDP Sciences, 2013

\title{
Direct drive fuel target optimization in HIF
}

\author{
S. Koseki ${ }^{1}$,a, S. Kawata ${ }^{1}$, Y. Hisatomi ${ }^{1}$, T. Kurosaki ${ }^{1}$, D. Barada ${ }^{1}$ \\ and A.I. Ogoyski ${ }^{2}$ \\ 1 Utsunomiya University, Utsunomiya 321-8585, Japan \\ ${ }^{2}$ Varna Technical University, Varna 9010, Bulgaria
}

\begin{abstract}
This study investigates a target for heavy ion fusion (HIF) using light target materials. In this study, the target structure, the heavy ion beam (HIB) input pulse shape and the HIB input pulse energy are optimized for the maximal fusion energy output. We performed two-dimensional fluid implosion simulations to obtain a high pellet gain. The optimized target shows a high yield of a gain 223 . The input $\mathrm{Pb}$ beam energy is $1.8 \mathrm{MJ}$.
\end{abstract}

\section{INTRODUCTION}

There are many research issues in heavy ion beam (HIB) inertial confinement fusion (ICF), such as beam bunching, HIB transport in a reactor, non-uniformity of beam irradiation, implosion uniformity, implosion efficiency, energy gain and so on. In target implosion, ICF has two ways of implosion schemes, that is, indirect-driven scheme and direct-driven implosion scheme [1-8].

In this paper, we employ a direct-drive scheme, and we present an improvement of the pellet gain by the optimization of the target structure and the HIB input pulse shape. As a result, we obtained a pellet gain of 223 by optimizing the target structure, the HIB input pulse shape and the HIB input pulse energy.

\section{OPTIMIZATION OF TARGET STRUCTURE AND HIB PULSE SHAPE}

Figure 1(a) shows the target in our previous work [9-11]. This target consists of $\mathrm{Pb}$ and $\mathrm{Al}$ as a tamper and a HIB-energy absorber, respectively [9-11]. We employed $32 \mathrm{~Pb} \mathrm{HIBs}$. The ion energy is $8 \mathrm{GeV}$ with the temperature of $100 \mathrm{MeV}$. The spot size of each HIB is $3.4 \mathrm{~mm}$, and the profile is a Gaussian one. The HIBs illumination scheme is presented in Ref. [10]. Our fluid implosion code is an ALE (Arbitrary Lagrangian and Eulerian) method with one radiation temperature diffusion model and a precise HIB ion energy deposition computation shown in Ref. [10]. In our 2D simulations in this paper, we performed simulations for the 90-degree section of the target. We introduce the precise HIB ion energy deposition in Ref. [2], and in this paper the nonuniformity introduced is about $2 \%$. The stopping power of a target is the sum of the energy deposited in target nuclei, target bound electrons, free electrons and target ions. In our present study, the target in Fig. 1(a) was designed to absorb about $30 \%$ of the input HIB energy. Figure 1(b) shows a lightened target, keeping the same thickness of the Al tamper. The target consists of $\mathrm{Al}$ and $\mathrm{C}$ as a tamper and a HIB-energy absorber, respectively [13]. Figure 1(c) shows the input pulse which we used for each target. We call the lower pulse power in the input pulse the foot pulse and the

\footnotetext{
${ }^{a}$ e-mail: kwt@cc.utsunomiya-u.ac.jp
}

This is an Open Access article distributed under the terms of the Creative Commons Attribution License 2.0, which permits unrestricted use, distribution, and reproduction in any medium, provided the original work is properly cited. 
(a)

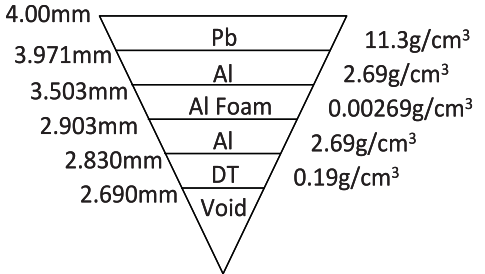

(b)

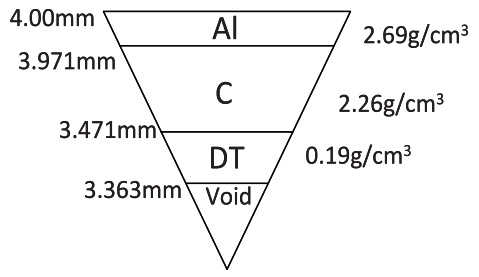

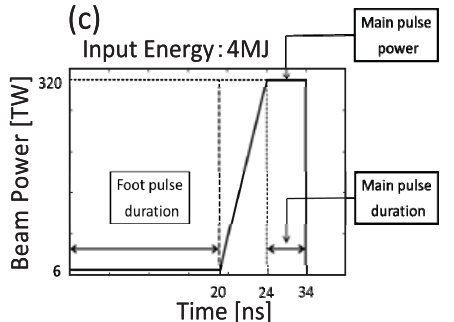

Figure 1. Target structures (a) consists of $\mathrm{Pb}$ and $\mathrm{Al}$ as a tamper and a HIB-energy absorber, respectively, and (b) consists of $\mathrm{Al}$ and $\mathrm{C}$ as a tamper and a HIB-energy absorber, respectively. (c) The HIB pulse shape used for each target.

(a)

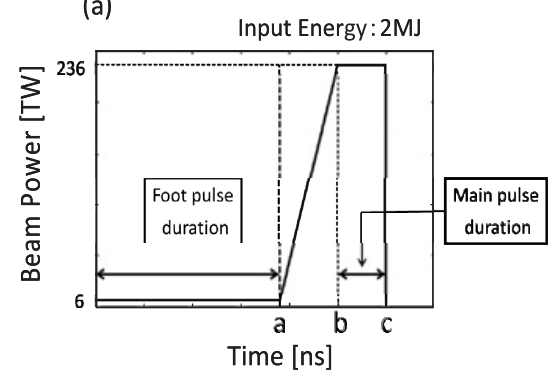

(b)

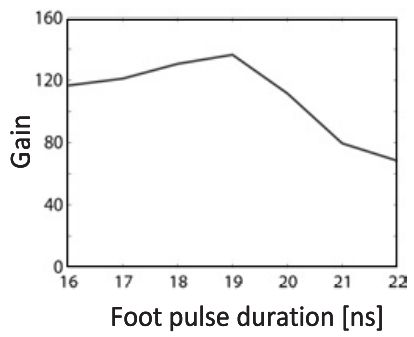

Figure 2. (a) The HIB pulse shape and (b) the pellet gain versus the foot pulse duration.

high pulse power in the input pulse the main pulse. The input $\mathrm{Pb}$ beam energy is $4 \mathrm{MJ}$. When the target in our previous work in Fig. 1(a) is used, we obtain a gain of 52.6. A higher gain of 61.9 can be achieved by the light-material of tamper (Al) and HIB-energy absorber (C).

The foot pulse duration is designed so that the main shock by the main pulse overlaps at the inner edge of the DT liquid fuel with the first weak shock to minimize the DT fuel preheating. Therefore, we perform a parameter study for the foot pulse duration for the light material target in Fig. 1(b). Figure 2(a) shows the input pulse shape and Fig. 2(b) shows the target gain when the foot pulse duration is changed. When the foot pulse duration is changed from $16 \mathrm{~ns}$ to $22 \mathrm{~ns}$, the irradiation for the main pulse starts after $2 \mathrm{~ns}$ of the foot pulse end. The HIB input energy is fixed to $2 \mathrm{MJ}$, and the main pulse duration is changed to keep the HIB total energy. The maximum gain is at $19 \mathrm{~ns}$ for the foot pulse duration (see Fig. 2(b)).

The effect of the thickness of energy absorber on the target gain is presented. Figure 3(a) shows the target structure, Fig. 3(b) the isentrope parameter in the DT fuel layer, and Fig. 3(c) the pellet gain versus the $\mathrm{C}$ absorber thickness. The absorber thickness is decided by changing the inner radius of the energy absorber layer. We vary the $\mathrm{C}$ absorber thickness from $400 \mu \mathrm{m}$ to $700 \mu \mathrm{m}$ and set the optimal foot pulse duration in the pulse shape for each thickness of the energy absorber. In addition, the irradiation for the main pulse starts after $2 \mathrm{~ns}$ of the foot pulse termination. The HIB input energy is fixed to $2 \mathrm{MJ}$, and the main pulse duration is changed to keep the HIB total energy (see Fig. 2(a)). When the C absorber thickness is small, preheating of the DT fuel layer becomes significant. However, when the $\mathrm{C}$ absorber thickness is large, the pellet gain becomes lower (see Fig. 3(b)). When the thickness of the energy absorber is $500 \mu \mathrm{m}$, we obtain the gain of 137 (see Fig. 3(c)).

The effect of the main pulse power in the input pulse shape on the target gain is presented. Figure 4(a) shows the input pulse shape. Figure 4(b) shows histories of the isentrope parameter in the DT fuel layer and Fig. 4(c) the average implosion velocity in the DT fuel layer for the main pulse powers of 
(a)

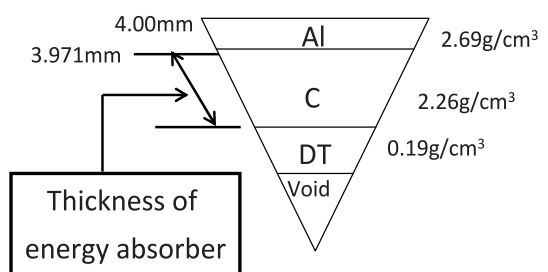

(b)

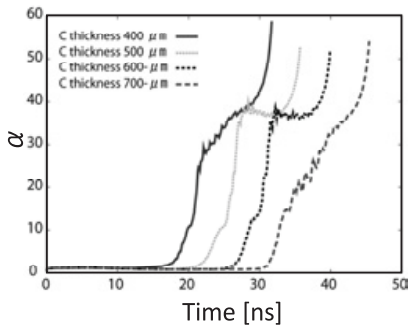

(c)

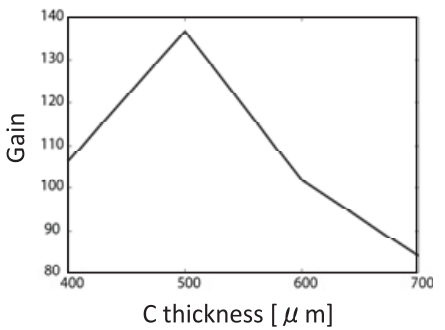

Figure 3. (a) Target structure, (b) histories of the isentrope parameter for the DT fuel layer in the thicknesses of energy absorber $400 \mu \mathrm{m}, 500 \mu \mathrm{m}, 600 \mu \mathrm{m}$ and $700 \mu \mathrm{m}$, and (c) the pellet gain versus the thickness of energy absorber.

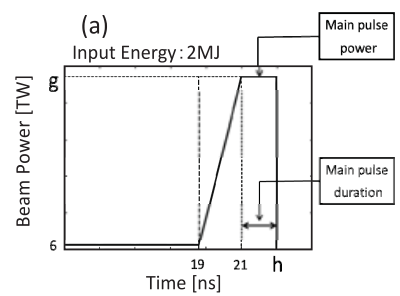

(b)

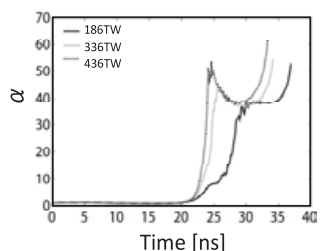

(c)

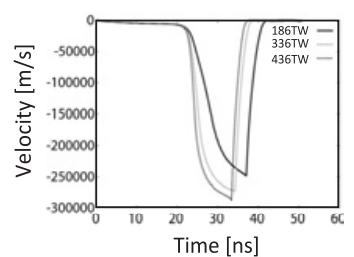

(d)

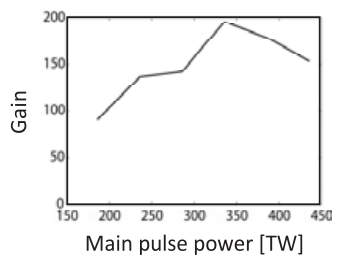

Figure 4. (a) The HIB pulse shape in the change of main pulse power, histories of (b) the isentrope parameter and (c) the average velocity for the DT fuel layer in the main pulse power $186 \mathrm{TW}, 336 \mathrm{TW}$ and $436 \mathrm{TW}$, and (d) the pellet gain versus the main pulse power.

$186 \mathrm{TW}, 336 \mathrm{TW}$ and $436 \mathrm{TW}$. Figure 4(d) shows the pellet gain versus the thickness of the main pulse power. We perform a parameter study for the main pulse power for Fig. 1(b). In Fig. 4(a), the foot pulse duration is $19 \mathrm{~ns}$, and the irradiation for the main pulse starts after $2 \mathrm{~ns}$ of the foot pulse termination. We vary the main pulse power from $186 \mathrm{TW}$ to $436 \mathrm{TW}$. The HIB input energy is fixed to $2 \mathrm{MJ}$, and the main pulse duration is changed to keep the HIB total energy. When the main pulse power is high, the preheating of the DT fuel layer becomes large (see Fig. 4(b)). When the main pulse power becomes low, the implosion speed of the DT fuel layer does not achieve a sufficient speed, also decreasing the target gain (see Fig. 4(c)). The maximal gain is obtained, when the main pulse power is $336 \mathrm{TW}$ (see Fig. 4(d)).

The effect of the HIB input energy on the target gain is presented. We perform a parameter study for the HIB input energy for Fig. 1(b). In the HIB pulse shape, the foot pulse duration is $19 \mathrm{~ns}$, and the irradiation for the main pulse starts after $2 \mathrm{~ns}$ of the foot pulse end. We vary the total energy from $1.4 \mathrm{MJ}$ to $2.0 \mathrm{MJ}$ in the pulse by varying duration of the main pulse, and holding the main pulse power of $336 \mathrm{TW}$ constant. Figure 5(a) shows the isentrope parameter in the DT fuel layer and Fig. 5(b) the average implosion velocity in the DT fuel layer for the HIB input energy of $1.4 \mathrm{MJ}, 1.8 \mathrm{MJ}$ and $2.0 \mathrm{MJ}$. Figure 5(c) shows the pellet gain versus the input energy. When the HIB input energy become large, the pre-heat in the DT fuel layer becomes significant (see Fig. 5(a)). When the HIB input energy is small, the efficient implosion is not possible, because the implosion velocity in the DT fuel layer does not become a sufficient speed (see Fig. 5(b)). As a result, the HIB input energy has an influence on the target gain. When the HIB input energy is 1.8 MJ, we obtain the gain of 223 (see Fig. 5(c)). 
(a)

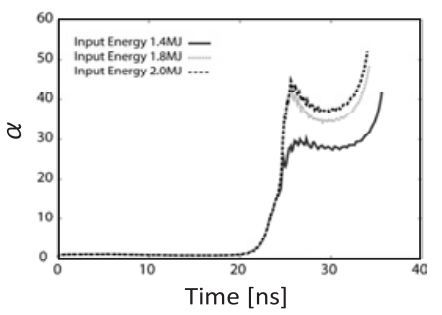

(b)

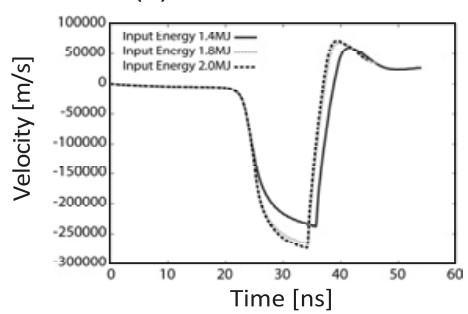

(c)

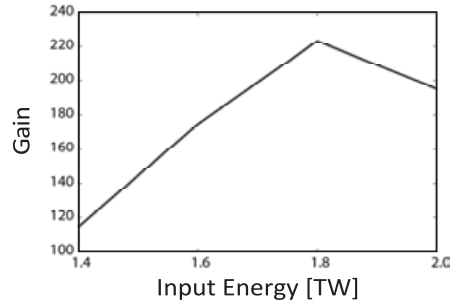

Figure 5. Histories of (a) the isentrope parameter and (b) the average velocity for the DT fuel layer in the total HIB input energy of $1.4 \mathrm{MJ}, 1.8 \mathrm{MJ}$ and $2.0 \mathrm{MJ}$, (c) the pellet gain versus the HIB input energy.

\section{CONCLUSIONS}

In this paper, we discussed the improvement of the pellet gain by the light material, the target structure, the optimizations of the HIB input pulse shape and the HIB input pulse energy. The optimized target structure consists of the $\mathrm{Al}$ thickness $30 \mu \mathrm{m}$ and the $\mathrm{C}$ thickness $500 \mu \mathrm{m}$ as a tamper and a HIB-energy absorber, respectively. In the optimized target, the DT fuel thickness is about $110 \mu \mathrm{m}$ and the void radius is $3.36 \mathrm{~mm}$. We obtain the higher gain in this study, compared with that for the heavy-material target (see Fig. 1(a)). In the near future, a robustness of the target implosion against the implosion nonuniformity should be studied for the target shown in Fig. 1(b).

This work was partly supported by the JSPS (Japan Society for the Promotion of Science), MEXT (Ministry of Education, Culture, Sports, Science and Technology) and CORE (Center for Optical Research and Education, Utsunomiya Univ., Japan). We would also like to present our thanks to colleagues in the Japan and US HIF VNL research groups for their fruitful discussions on this subject.

\section{References}

[1] C. K. Li, F. H. Seguin, J. A. Frenje, R. D. Petrasso, Phys. Rev. Lett. 92, 205001 (2004)

[2] M. Tabak, D. Callahan-Miller, Phys. Plasmas 5, 1895 (1998)

[3] D. A. Callahan, Appl. Phys. Lett. 67, L3254 (1995)

[4] M. Tabak, D. Callahan-Miller, D. D. M. Ho, G. B. Zimmerman, Nucl. Fusion 38, 509 (1998)

[5] S. H. Glenzer, L. J. Suter, R. E. Turner, B. J. MacGowan, K. G. Estabrook, M. A. Blain, S. N. Dixit, B. A. Hammel, R. L. Kauffman, R. K. Kirkwood, O. L. Landen, M. C. Monteil, J. D. Moody, T. J. Orzechowski, D. M. Pemmimgton, G. F. Stone, T. L. Weiland, Phys. Rev. Lett. 80, 2845 (1998)

[6] M. D. Cable, S. P. Hatchett, J. A. Caird, J. D. Kilkenny, H. N. Kornblum, S. M. Lane, C. Laumann, R. A. Larche, T. J. Murphy, J. Murray, M. B. Nelson, D. W. Phillion, H. Powell, D. B. Ress, Phys. Rev. Lett. 73, 2316 (1994)

[7] Y.Y. Lau, Simon S. Yu, John J. Barnard and Peter A. Seidl, Laser and Particle Beams 27, 279 (2011)

[8] H. Qin, R. C. Davidson and B. G. Logan, Laser and Particle Beams 27, 365 (2011)

[9] S. Kawata, K. Miyazawa, T. Kikuchi, T. Someya, Nuclear Instrument and Methods in Physics Research A 577, 332 (2007)

[10] K. Miyazawa, A. I. Ogoyski, S. Kawata, T. Someya, T. Kikuchi, Phys. Plasmas 12, 122702 (2005)

[11] S. Kawata, K. Miyazawa, A. I. Ogoyskii, T. Kikuchi, Y. Akasaka, Y. Iizuka, Journal of Physics: Conference Series 112, 032028 (2008)

[12] T. Someya, K. Miyazawa, T. Kikuch, S. Kawata, Laser and Particle Beams 24, 359-369 (2006)

[13] B. G. Logan, L. J. Perkins, J. J. Barnard, Phys. Plasmas 15, 072701 (2008) 